\title{
Renal Radionuclide Studies
}

\author{
Keith Britton
}

Our adventure with the kidneys started at the then Middlesex Hospital culminating in 1971 with Britton and Brown's monograph Clinical Renography [1]. This was prefaced: 'The disciplines of Medicine and Physics are like oil and vinegar - often shaken together, often appetising but not easily miscible'. We attempted to 'blend the soothing oil of medicine with the acid reality of Physics in a study of common interest: how the kidney works'. We debunked the old nomenclature of the renogram - the Vascular spike, the Secretory phase and the Excretory phase - for example the latter is the 'left behind in the kidney' phase. We proposed 'Second phase' for the rising portion and 'Third phase' for the descending portion of the renal activity time curve. We cleaned up the renogram with Computer assisted blood background subtracted, CABBS, Renography [2]. With a grant from the Leverhulme Trust, we developed a mobile Renography trolley and couch with Nuclear Enterprises of Edinburgh, which could be taken to the Intensive care unit and the patient transferred onto it.

NJG, Nick, Brown first showed the importance of the integral of the Blood clearance curve [1,3], later used as the Patlak or Rutland plot (Mike Rutland did his MSc with us). It led to the numerical approach to measuring the Outflow Efficiency. Nick also developed various renal transit times.

K. Britton

Departments of Radiology and Nuclear Medicine, The London Clinic,

20 Devonshire Place, London W1G 6BW, UK 


\subsection{Essential Hypertension}

Using the bimodal distribution of transit times, we were able to separate the contributions of the Cortical and Juxta-medullary nephrons to renal blood flow [4]. These were validated in animal studies with Steve Wilkinson at King's College. We were able to show that in Essential Hypertension, Cortical blood flow is reduced and Medullary blood flow is increased [5], the former increasing salt retention and the latter accounting for loss of urine concentrating ability, Nocturia being a common symptom of early Hypertension. We were also able to show that Captopril greatly improved Cortical nephron flow and reduced blood pressure. It led to Lancet publications of the hypothesis 'Renin and Renal Autoregulation' [6] and 'Essential Hypertension: a disorder of cortical nephron control' [7].

\subsection{Measurement Based Medicine}

These approaches were based on the premise that numerical indices of renal radionuclide studies were better than eye-balling and guessing the meaning of a complex activity time curve. Indeed our philosophy became a wish to substitute 'Evidence based medicine' which applied to the many with 'Measurement based medicine' which applied to the individual through 'Normal ranges' and 'Action determining ranges'. For example in adults one should act to save a kidney with more than $15 \%$ of total renal function, but not if it is less that $5 \%$ of total. Sreenevasan's dictum was made during my IAEA time in Malaysia 'In the presence of bilateral outflow obstruction (usually uric acid stones) always operate on the kidney with the better relative function first as determined by renography' [8].

The refinements occurred at St Bartholomew's Hospital in the City of London through the Physicist Cyril Nimmon's expertise and dedication. The Gamma camera replaced the probes and his advanced computer programmes enhanced and simplified the results [9].

\subsection{Outflow Efficiency}

The Outflow efficiency [10] is determined by subtracting the renal activity time curve from the integral of the blood clearance curve (the input curve) fitted to its second phase to give the integral of the output curve. This output curve is then taken as a percentage of the input curve. Unlike Frusemide dependent measurements, it is independent of the level of renal function. Normally a kidney should excrete over $78 \%$ of what it took up within $30 \mathrm{~min}$.

\subsection{Transit Times}

Renal Transit times seem to confuse some practitioners. Think of 'Pooh sticks'. On one side of the bridge a bunch of sticks are thrown into the stream together. You run to the other side of the bridge and see one stick coming out first followed by a 
distribution of others. The distribution of times they take to pass under the bridge can be measured and a mean time of transit determined. The mathematical process is Deconvolution. The result tends to be noisy. Three factors made it usable. Firstly the high count rate given by Tc-99m MAG3. This was first used in the UK in our department and published by Dr now Professor Adil Al Nahhas [11]. Secondly Cyril Nimmon devised two constraints on the activity time curve, monotonicity and non-negativity; and a lack of over-smoothing of the original data; thirdly the use of the left ventricle to give the blood clearance curve as a high count input.

The understanding of Transit times by the clinician may be likened to learning to ride a bicycle. One falls off several times at the start and either gives up as many have; or persists until one avoids the potholes and is rewarded by the ride.

\subsection{Renovascular Disorders}

It may be noted that renal radionuclide studies do not generally distinguish between large and small vessel renal disease if they are functionally significant in contributing to hypertension. The Mean Parenchymal Transit Time, MPTT is normally less than $240 \mathrm{~s}$. In the context of Hypertension due to Renovascular disorder such as Renal artery stenosis, it exceeds this and indicates the narrowing is functionally significant. It is further increased in this context by Captopril, particularly in large vessel disease. Gruenewald et al. [12], who did his MSc with us, showed that if MPTT was prolonged then most hypertensive patients are benefitted by revascularisation or stenting with a reduction of their Blood Pressure, but if MPTT was normal in this context, none of the Hypertensive patients benefitted.

\subsection{Obstructing Uropathy}

Consider some basics, there is the Force from cardiac output, which does not change if there is a stone blocking urine flow. There is the Resistance to urine flow. The consequence of the interaction of a Force and a Resistance is a rise in Pressure. Pressure is not a cause but a consequence. Pressure is equal and opposite in all directions (Sir Isaac Newton) so there is no such thing as 'back pressure'. When there is a system of fluid in a tube, it flows from the higher pressure to the lower pressure. A non reabsorbable agent such as Tc-99m MAG3 therefore takes time to travel down this pressure gradient. In Obstructing Uropathy, the resistance to flow increased so the solute takes longer to transit. Increased resistance to flow is thus transmutable into an increased transit time which can be measured. In this context the Parenchymal Transit Time Index, PTTI is helpful. It is measured as the MPTT minus the Minimum (shortest) Transit time which makes an allowance for different urine flow rates. Normal PTTI is less than $156 \mathrm{~s}$. Unlike Frusemide based studies, it is independent on the level of renal function, although there has to be sufficient function for the measurement to be made. The Urologist Mr Hugh Whitfield at St Barts gained the Hunterian award for evaluating the advantages of using PTTI in the context of potential Obstructing Uropathy [13, 14]. An abnormal PTTI is one of the earliest indicators of functionally significant Obstructing Uropathy in adults, for example when renal function is still normal. It is 
also independent of the size of the renal pelvis. If the words 'outflow obstruction' or worse 'partial obstruction' were replaced by 'increased resistance to flow', the understanding of an 'outflow obstruction' would be made easier.

The above is a simplification of Transit time usage, which needs some interpretive skill, for example PTTI may be unreliable if MPTT is abnormal. Transit time indices should be taken together with other clinical information and renal measurements in a holistic approach.

\subsection{Frusemide Response}

There are a number of ways of evaluating the response to Frusemide. Visually one may compare the rising second phase with the falling third phase of the activity time curve. If the rate of fall of the third phase before or after Frusemide is appropriate to the rate of rise of the second phase, then no Obstructive Nephopathy is likely. However using a T1/2 or other measure of the rate of fall of the third phase will be normal if renal function is good and abnormal if renal function is poor. It is not to be relied upon. Our usual protocol is to inject Frusemide at 16 min $(\mathrm{F}+16)$ so that one can see the visual effect of Frusemide on any pelvic retention of tracer and its effect on the third phase of the activity time curve. When Frusemide is given 15 min before the start of the renal activity time curve, (F-15), which was previously used when the renogram was equivocal, the response to Frusemide cannot be assessed, except by comparison with a previous study without F-15. F-15 often causes the patient urinary urgency and may cause early termination of the study.

The numerical indices Outflow efficiency [15] and Parenchymal transit time index [16] avoid the need for F-15 in equivocal studies.

Whereas MAG3 is anionically bound, Cyclosporine is cationically bound in the kidney, hence the lack of reliability of Tc-99mMAG3 in this context of renal transplantation. Ajit Padhy and Kishor Solanki developed a new cationic binding agent Tc-99m DACH intended for the evaluation of cyclosporine toxicity [17]. Jamshed Bomanji among many other contributions showed the early beneficial effects of Lithotripsy on the outcome of the treatment of renal calculi [18]. We developed a 'bootstrap' model of the 'Counter Current' mechanism of urinary concentration with our systems analysis colleagues from City University [19]. In conclusion we emphasised that Nuclear Medicine allowed the appreciation and measurement of renal physiology and patho-physiological processes in man.

Since this section was to be related mainly to the work at St Bartholomew's Hospital, I apologize for being unable to mention the works of many others in renal function measurements, renal transplants, renal tumours and Paediatrics. With their help, Renal Radionuclide studies have an established place in Nephrology and Urology. 
Open Access This chapter is distributed under the terms of the Creative Commons AttributionNoncommercial 2.5 License (http://creativecommons.org/licenses/by-nc/2.5/) which permits any noncommercial use, distribution, and reproduction in any medium, provided the original author(s) and source are credited.

The images or other third party material in this chapter are included in the work's Creative Commons license, unless indicated otherwise in the credit line; if such material is not included in the work's Creative Commons license and the respective action is not permitted by statutory regulation, users will need to obtain permission from the license holder to duplicate, adapt or reproduce the material.

\section{References}

1. Britton KE, Brown NJG. Clinical renography. London: Lloyd Luke; 1971.

2. Britton KE, Brown NJG. Computer Assisted Blood Background Subtraction (CABBS) renography in obstructive nephropathy. Proc Roy Soc Med. 1970;63:1246-7.

3. Brown NJG, Britton KE. The renogram and its quantitation. Br J Urol. 1969;41(Suppl):15-25.

4. Britton KE. The measurement of intrarenal blood flow distribution in man. Clin Sci Mol Med. 1979;56:101-4.

5. Gruenewald SM, Nimmon CC, Nawaz MK, Britton KE. A non-invasive gamma camera technique for the measurement of intrarenal flow distribution in man. Clin Sci (London). 1981;61:385-9.

6. Britton KE. Renin and renal autoregulation. Lancet. 1968;2:329-33.

7. Britton KE. Essential hypertension: a disorder of cortical nephron control? Lancet. 1981;ii:900-2.

8. Sreenevasan G. Bilateral renal calculi. Ann Roy Coll Surg Engl. 1974;55:3-12.

9. Britton KE, Brown NJG, Nimmon CC. Clinical renography: 25 years on. Eur J Nucl Med. 1996;23:1541-6.

10. Chaiwatanarat T, Padhy AK, Bomanji JB, et al. Validation of renal output efficiency as an objective quantitative parameter in the evaluation of upper urinary tract obstruction. J Nucl Med. 1993;34:845-8.

11. Al Nahhas AA, Jafri RA, Britton KE, et al. Clinical experience with 99mTc-MAG3 mercaptoacetyl triglycine and a comparison with 99mTc-DTPA. Eur J Nucl Med. 1988;14:453-62.

12. Greuenewald SM, Stewart JH, Simmons KC, Crocker EF. Predictive value of quantitative renography for successful treatment of atherosclerotic renovascular hypertension. Aust $\mathrm{N} \mathrm{Z}$ J Med. 1985;15:617-22.

13. Whitfield HN, Britton KE, Nimmon CC, Hendry WF, Wallace DM, Wickham JE. Renal transit time measurements in the diagnosis of ureteric obstruction. Br J Urol. 1981;53:500-3.

14. Britton KE, Nimmon CC, Whitfield HN, et al. Obstructive nephropathy, successful evaluation with radionuclides. Lancet. 1979;1:905-7.

15. Cosgriff PS, Morrish O. The value of output efficiency measurement in resolving equivocal Frusemide responses. Nucl Med Commun. 2000;21:390-1.

16. Britton KE, Nawaz MK, Whitfield HN, et al. Obstructive nephropathy: comparison between Parenchymal transit time index and Frusemide diuresis. Br J Urol. 1987;59:127-32.

17. Padhy AK, Solanki KK, Bomanji J, et al. Clinical evaluation of Tc-99m Diamino-cyclohexane, a renal agent with cationic transport: results in healthy normal volunteers. Nephron. 1993;65:294-8.

18. Bomanji J, Boddy SAM, Britton KE, et al. Radionuclide evaluation of pre- and postextracorporeal shock wave lithotripsy for renal calculi. J Nucl Med. 1987;28:1284-9.

19. Britton KE, Cage PE, Carson ER. A 'bootstrap' model of the renal medulla. Postgrad Med J. 1976;52:279-84. 


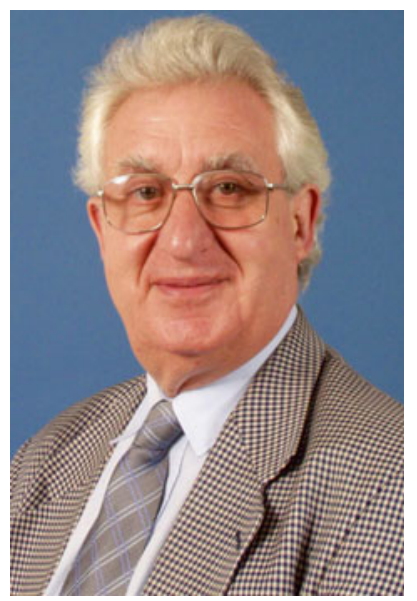

Keith Britton I was educated at Malvern College, Downing College, Cambridge and the then Middlesex Hospital, London. I was registrar to Dr John Nabarro and Dr 'Willy' Slater and carried out the hospital's first renal dialysis. I became fascinated with Nuclear Medicine as a non-invasive tool to study how organs functioned in people and not just their anatomy. I studied with Prof Edward Williams at the Institute of Nuclear Medicine, Middlesex Hospital gaining the MSc; and an MD at Cambridge examined by Professor Norman Veall. He said that he had repeated my work on renal transit times and agreed with it. That was the exam! I became registrar to Professor Stanley Peart at St Mary's Hospital, learning about the management of Hypertension. This prepared me for my part in the Hypertension Clinic at St Bartholomew's Hospital. I was appointed as a General Physician at St Barts. If the clinic staff could not decide on the specialty to which a GP had referred a patient, then it would go to me as a generalist to sort out in the outpatient clinic.

I then became a Professor of Nuclear Medicine at the Medical school and Queen Mary College, giving up my ward duties at St Barts. I was made director of the Nuclear Medicine group of Cancer Research UK, which funded staff and equipment to carry out research into imaging and therapy with radio-labelled monoclonal antibodies in cancers. During my time and work with the International Atomic Energy Agency, we were able to welcome over 100 overseas postgraduate doctors to sit the MSc in Nuclear Medicine, most passed. As a past President of the Society of Nuclear Medicine, Europe, I was able to help to found the European Association of Nuclear Medicine, EANM and lead the congress in 1985 at the Barbican centre in London.

I was a past President of the BNMS and organised that Physicists became full members. I was one of the founder editors of Nuclear Medicine Communications and of the World Journal of Nuclear Medicine. I published over 250 peer reviewed manuscripts and several books including Clinical Nuclear Medicine, lead Editor Professor Michael Maisey, going on for four editions.

After my retirement due to age 65, I was awarded Emeritus Professor, University of London. I became Chair of the Board of the independent Cromwell Hospital for two and a half years. It was revealing to be able to manage instead of being managed by the NHS. I continue in private practice as Consultant in Nuclear Medicine to The London Clinic and the London Bridge Hospital. 